\title{
A decrease in gamma-synuclein expression within the nucleus accumbens increases cocaine intravenous self-administration in the rat
}

\author{
Frédéric Boyer', Eric Balado ${ }^{2,3}$, Pier V. Piazza ${ }^{2,3}$, Jean-Luc Dreyer' \& \\ Véronique Deroche-Gamonet ${ }^{2,3}$
}

Institute of Biochemistry, University of Fribourg, Fribourg, Switzerland', INSERM U862, NeuroCentre Magendie, Pathophysiology of Addiction, Bordeaux, France ${ }^{2}$ and Université de Bordeaux, Bordeaux, France ${ }^{3}$

\begin{abstract}
Except as a marker of cancer progression, gamma-synuclein (GSyn) had received little attention. Recent data showed however that GSyn modulates cocaine-induced locomotor effects, suggesting that it could also play a role in cocaine reinforcing effects. In the rat, siRNAs targeting GSyn expression were injected in the nucleus accumbens and cocaine reinforcing effects were evaluated by means of intravenous self-administration. A dose-response curve was followed by procedures of progressive ratio, extinction, cocaine- and cue-induced reinstatements. Decrease of GSyn expression increased self-administration over a large range of doses. This effect was associated with an increase in cocaine-induced reinstatement. The present data reveal that GSyn exert a specific negative control on cocaine-induced reinforcing and incentive effects.
\end{abstract}

Keywords Cocaine, gamma-synuclein, incentive, intravenous self-administration, rat, siRNA.

Correspondence to: Véronique Deroche-Gamonet, INSERM U862, NeuroCentre Magendie, Pathophysiology of Addiction, 146 Rue Léo Saignat, 33077 Bordeaux Cedex, France. E-mail: veronique.deroche@inserm.fr

Synucleins are three soluble proteins expressed in neural tissue (Surguchov 2008): alpha- (Asyn), beta- and gamma-synuclein (GSyn). Consistent with its role in dopamine homeostasis (Xu et al. 2002), ASyn has been unsurprisingly associated with drug use-related disorders (Liang et al. 2003; Mash et al. 2003; Bonsch et al. 2004). GSyn is distinct from the two other family members in terms of structure and cell localization and has been mainly characterized as a marker of cancer progression (Surguchov 2008). Recent data, however, revealed that GSyn can modulate dopaminergic activity (Moszczynska et al. 2007) and cocaine-induced locomotor effects (Boyer \& Dreyer 2008). We therefore hypothetized that GSyn might also modulate cocaine renforcing effects and therefore cocaine use.

In the rat, lentiviruses driving the expression of small interfering RNAs against GSyn mRNA (LV-GSyn-siRNAs) were administered in the nucleus accumbens, a key structure mediating cocaine-induced reinforcing effects (Kalivas, Volkow \& Seamans 2005). Cocaine use was evaluated using intravenous self-administration (SA).
siRNAs targeted against GSyn mRNA or Green Fluorescent Protein mRNA (LV-GFP, control) were produced (Bahi et al. 2004a; Bahi et al. 2004b; Boyer \& Dreyer 2008) and administered using a classical stereotaxic approach. In the same time, rats were equipped with an intravenous catheter. Seven days later, rats were trained to self-administer cocaine at a dose of $0.8 \mathrm{mg} / \mathrm{kg} /$ infusion for 12 days (see Appendix S1 in the Supporting Information for details on the experimental SA set-up and procedures). During the acquisition phase, stability in drug intake (Fig. 1a) and preference for the active device (a hole in which nosepoking induces cocaine delivery), against the inactive one [hole, $\mathrm{F}(1,15)=35.2, P<0.0001]$ (data not shown), attested to the fact that cocaine was self-administered. During this acquisition period, LV-GSyn-siRNAs did not alter cocaine intake, total responding or discrimination between active and inactive devices.

A dose-response curve was then performed. Both the dose-response functions for cocaine intake [dose, $\mathrm{F}(4,64)=22.06, \quad P<0.0001$; group effect, $\mathrm{F}(1,16)=$ 5.618, $\quad P<0.05, \quad$ group $\times$ dose, $\quad \mathrm{F}(4,64)=1.721$, 
Figure I Acquisition (a), and doseresponse curve $(b-c)$, of cocaine selfadministration in rats administered with LV-GSyn-siRNAs (grey circles and squares) or LV-GFP (controls, white circles and squares) in the nucleus accumbens. Nosepokes into active (delivering cocaine) and inactive (control) holes were used as operant responses to evaluate selfadministration. (a) Self-infusions $(0.8 \mathrm{mg} / \mathrm{kg} /$ infusion) as a function of response requirement (FR) during acquisition. Each point represents the mean sum of selfinfusions ( \pm SEM) per self-administration session. (b-c). Self-infusions and responses in the active (circles) and inactive (squares) holes as a function of cocaine dose. Each point represents the mean sum ( \pm SEM) per session over the last two sessions for each dose.
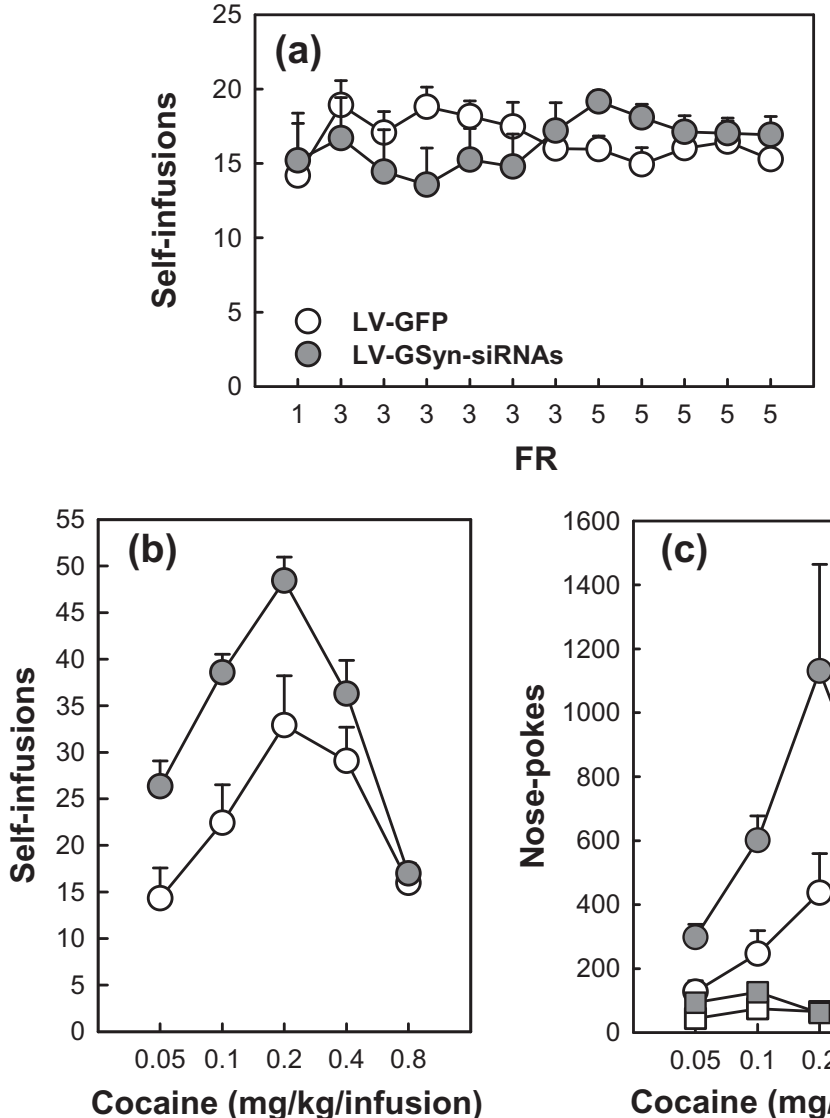

$P=0.15]$ and active responses [dose, $\mathrm{F}(4,64)=13.16$, $P<0.0001$; group, $\mathrm{F}(1,16)=4.03, P<0.05$, groupxdose, $\mathrm{F}(4,64)=0.69, P=0.60]$ were shifted upward in LV-GSyn-siRNAs rats (Fig. 1b \& c). Whatever the cocaine dose, inactive responses were similar in the two groups, suggesting that LV-GSyn-siRNAs treatment produced a specific increase in cocaine reinforcing effects.

To fully characterize the psychopharmacological consequences of LV-GSyn-siRNAs, we then performed: (1) a progressive ratio schedule evaluating motivation for the drug (Richardson \& Roberts 1996); (2) an extinction procedure, during which the drug is withdrawn and responding is without scheduled consequences (Extinction is considered as a new learning resulting in an active inhibitory control over the learned operant behavior (Bouton 2004; Woods \& Bouton 2007). The extinction procedure allows then measuring the ability to gain control over the behavioral response previously leading to cocaine infusions]; and (3) cocaine- and cue-induced reinstatements (Shaham \& Hope 2005). Small amounts of the abused drug, and drug-associated contextual cues, are factors known to possess incentive effects which can precipitate drugseeking (Shaham \& Hope 2005).

Whatever the dose of cocaine tested, LV-GSyn-siRNAs treatment highly tended to increase motivation for cocaine as measured by the breakpoint (BP; last ratio completed) in the PR schedule (Fig. 2a) $[\mathrm{F}(1,15)=1.85$, $P=0.18]$. On the contrary, the two groups did not differ for responding on both active and inactive devices during extinction (Fig. 2b).

Contingent presentations of the cocaine-associated cue light produced a significant reinstatement of the extinguished SA behavior (Fig. 2c and Appendix S2 in the Supporting Information) as attested by a higher number of responses in the active than in the inactive hole [hole, $\mathrm{F}(1,15)=12.09, P<0.005$ ]. This effect was, however, similar in both experimental groups.

Cocaine produced a dose-dependent $[$ dose, $\mathrm{F}(3,45)=$ 9.32, $P<0,0001]$ and specific [hole, $\mathrm{F}(1,15)=10.16$, $P<0.01 ;$ holexdose, $\quad \mathrm{F}(3,45)=7.08, \quad P<0.0005]$ reinstatement of the SA behavior (Fig. 2e). Indeed, responding in the inactive hole was not altered by cocaine and responding in both holes was not altered by vehicle infusions in the same experimental conditions (Fig. 2d, and Appendix S2 in the Supporting Information). Interestingly, cocaine-induced reinstatement was higher in LV-GSyn-siRNAs rats as compared with LV-GFP rats [groupxdose, $\mathrm{F}(3,45)=2.92, P<0.05$ ] and this effect was specific of the active hole [groupxdosexhole, $\mathrm{F}(3,45)=2.51, P<0.05]$. 

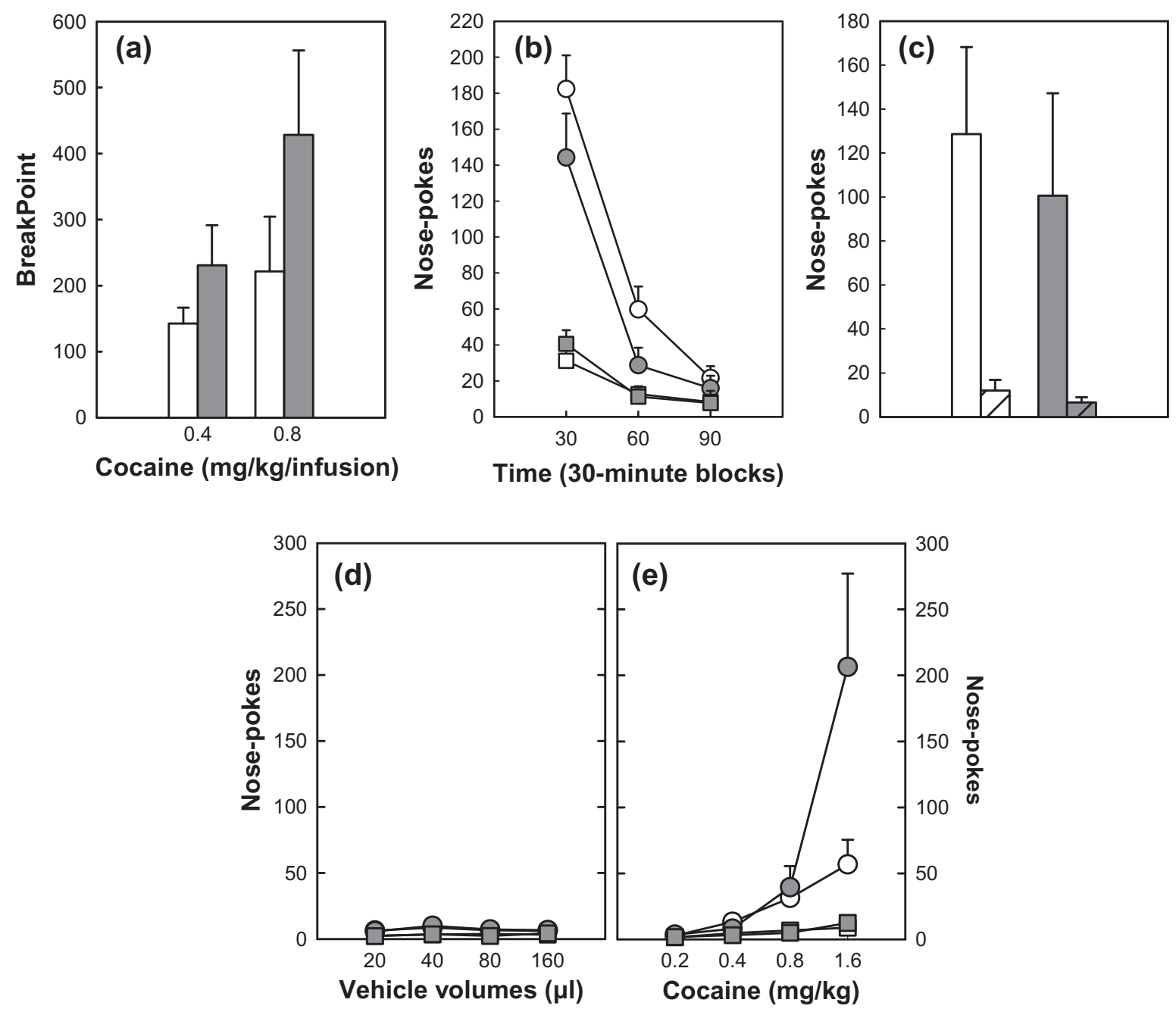

Figure 2 Differences between rats administered with LV-GSyn-siRNAs (in grey) and rats administered with LV-GFP (controls, in white) in the nucleus accumbens in: (a) Progressive ratio (PR). Motivation for cocaine was estimated as a function of cocaine dose by measuring the last ratio completed (Breakpoint: BP). The PR was applied over two consecutive sessions for each cocaine dose. Each bar represents the mean BP ( \pm SEM) over the last session. (b) Extinction. Each point represents the mean sum of responses ( \pm SEM) over 30-minute time blocks (circles and squares for active and inactive holes, respectively). (c) Cue-induced reinstatement. Each bar represents the mean sum of nose-pokes ( \pm SEM) over the 60 minutes of the contingent cue presentation (open and dashed bars for active and inactive holes, respectively). ( $d-e$ ) Vehicle- and cocaine-induced reinstatement. Each point represents the mean sum of responses ( \pm SEM) in the active (circles) and inactive (squares) holes over the 30 minutes following each vehicle or cocaine infusion (circles and squares for active and inactive holes, respectively).

Rats were killed 24 hours after the last SA session and nucleus accumbens sampled. GSyn transcripts were quantified by quantitative real-time PCR (qPCR). GSyn and Asyn proteins were quantified by Western blots (see details in Appendix S1 in the Supporting Information). Animals injected with LV-GSyn-siRNAs displayed a 50\% decrease in GSyn mRNA (Fig. S1A) and GSyn protein levels (Fig. S1B). The observed behavioral effects of LV-GSyn-siRNAs administration were not due to compensation in ASyn expression. ASyn protein levels in the two experimental groups (Fig. S1C) were comparable confirming previous data (Boyer \& Dreyer 2008).

Recent data suggested that GSyn might play a role in cocaine reinforcing effects (Boyer \& Dreyer 2008). By using siRNAs, we decreased GSyn expression in the nucleus accumbens, a brain structure thought to be a key actor for cocaine use, abuse and addiction (Kalivas et al. 2005). This manipulation increased cocaine SA. This increase was specifically associated with increased cocaine-induced incentive effects and a high trend to an increased motivation. It did not alter behavioral disinhibition as measured in an extinction procedure and cueinduced incentive effects as measured in cue-induced reinstatement. GSyn may exert a specific negative control on cocaine reinforcing and incentive effects in the nucleus accumbens.

In the present work, silencing of GSyn was shown to increase cocaine reinforcing and incentive effects, while 
the same manipulation was previously shown to decrease cocaine-induced locomotion (15 mg/kg) (Boyer \& Dreyer 2008). Further investigations are needed to explain what appears as a discrepancy. Since only one dose of cocaine was tested on locomotor activity, the decreased response could indeed reflect an increased cocaine efficacy expressed by increased stereotyped behaviors.

Neurobiological mechanisms underlying the modulation of cocaine reinforcing effects by GSyn have now to be investigated. Modulation of the DAT function might be a target of interest as GSyn has been shown in vitro to directly interact with the DAT and disrupt the ASyn-DAT interaction (Moszczynska et al. 2007). This mechanism fits with the effect of the combined manipulations of the DAT and GSyn levels on cocaine locomotor effects (Boyer \& Dreyer 2008).

\section{Acknowledgements}

This work was supported by the following grants: ANRAddiction (2005) and EU-STREP-PheCOMP (FP6) (PVP), MILDT/INCa/Inserm (2008) (VDG), Swiss National Foundation grants 3100-059350 and 3100AO-100686 (JLD). The authors are very grateful to Mr V. Chandrasekar and Mrs C. Deforel-Poncet for their skilful assistance. The authors report no financial conflict of interest.

\section{Authors contribution}

VDG and JLD designed the study. FB and EB performed the experiments. VDG supervised the study and wrote the article. VDG and FB analyzed the data. VDG, JLD and PVP provided funds. All authors have critically reviewed content and approved final version submitted for publication.

\section{References}

Bahi A, Boyer F, Gumy C, Kafri T, Dreyer JL (2004a) In vivo gene delivery of urokinase-type plasminogen activator with regulatable lentivirus induces behavioural changes in chronic cocaine administration. Eur J Neurosci 20:3473-3488.

Bahi A, Boyer F, Kafri T, Dreyer JL (2004b) CD81-induced behavioural changes during chronic cocaine administration: in vivo gene delivery with regulatable lentivirus. Eur J Neurosci 19:1621-1633.

Bonsch D, Reulbach U, Bayerlein K, Hillemacher T, Kornhuber J, Bleich S (2004) Elevated alpha synuclein mRNA levels are associated with craving in patients with alcoholism. Biol Psychiatry 56:984-986.
Bouton ME (2004) Context and behavioral processes in extinction. Learn Mem 11:485-494.

Boyer F, Dreyer JL (2008) The role of gamma-synuclein in cocaine-induced behaviour in rats. Eur J Neurosci 27:29382951.

Kalivas PW, Volkow N, Seamans J (2005) Unmanageable motivation in addiction: a pathology in prefrontal-accumbens glutamate transmission. Neuron 45:647-650.

Liang T, Spence J, Liu L, Strother WN, Chang HW, Ellison JA, Lumeng L, Li TK, Foroud T, Carr LG (2003) alpha-Synuclein maps to a quantitative trait locus for alcohol preference and is differentially expressed in alcohol-preferring and -nonpreferring rats. Proc Natl Acad Sci U S A 100:46904695.

Mash DC, Ouyang Q, Pablo J, Basile M, Izenwasser S, Lieberman A, Perrin RJ (2003) Cocaine abusers have an overexpression of alpha-synuclein in dopamine neurons. J Neurosci 23:25642571.

Moszczynska A, Saleh J, Zhang H, Vukusic B, Lee FJ, Liu F (2007) Parkin disrupts the alpha-synuclein/dopamine transporter interaction: consequences toward dopamine-induced toxicity. J Mol Neurosci 32:217-227.

Richardson NR, Roberts DC (1996) Progressive ratio schedules in drug self-administration studies in rats: a method to evaluate reinforcing efficacy. J Neurosci Methods 66:1-11.

Shaham Y, Hope BT (2005) The role of neuroadaptations in relapse to drug seeking. Nat Neurosci 8:1437-1439.

Surguchov A (2008) Molecular and cellular biology of synucleins. Int Rev Cell Mol Biol 270:225-317.

Woods AM, Bouton ME (2007) Occasional reinforced responses during extinction can slow the rate of reacquisition of an operant response. Learn Motiv 38:56-74.

Xu J, Kao SY, Lee FJ, Song W, Jin LW, Yankner BA (2002) Dopamine-dependent neurotoxicity of alpha-synuclein: a mechanism for selective neurodegeneration in Parkinson disease. Nat Med 8:600-606.

\section{SUPPORTING INFORMATION}

Additional Supporting Information may be found in the online version of this article:

Appendix S1 Supplementary Methods Appendix S2 Supplementary Results

Figure S1 Gamma- and alpha-synuclein expression in the nucleus accumbens of rats administered with LV-GSynsiRNAs (grey bars) and rats administered with LV-GFP (controls, white bars).

Please note: Wiley-Blackwell are not responsible for the content or functionality of any supporting materials supplied by the authors. Any queries (other than missing material) should be directed to the corresponding author for the article. 